\title{
Evolution de l'occupation des sols dans la partie Nord du Dallol Bosso, départements de Filingué et Balleyara, région de Tillabéri-Niger
}

\begin{abstract}
Issoufou Maigary
Département Information et Recherche du Centre Régional AGRHYMET, Niamey, Niger, Département de géologie de la FAST de l’Université Abdou Moumouni de Niamey, Niger
\end{abstract}

Boureïma Ousmane

Département de géologie de la FAST

de l'Université Abdou Moumouni de Niamey, Niger

Ado Dankarami

Département Information et Recherche

du Centre Régional AGRHYMET, Niamey, Niger

Doi: 10.19044/esj.2018.v14n30p391 URL:http://dx.doi.org/10.19044/esj.2018.v14n30p391

\begin{abstract}
The departments of Filingué and Balleyara, which are our study area, are located in the northern part of Dallol Bosso, Tillabéri region in western Niger. This study area is circumscribed between $13^{\circ} 35^{\prime}$ and $14^{\circ} 40^{\prime}$ north latitudes and $2^{\circ} 50$ 'and $3^{\circ} 30^{\prime}$ East longitude. The effects of climate variability and change in the region since the 1970s have had significant impacts on ecosystems. This paper focuses on analyzing the dynamics of land use land cover in that area. The methodology based on the interpretation of the satellite image for 1972, 1987 and 2016 has led to important results. Thus, there is a notable decline in areas covered by natural plant formations (tiger bush and steppe). Indeed, they range from $28.79 \%$ in 1972 to $12.15 \%$ in 2016 of the total surface area of the study area. However, farmland increased from 164772 ha in 1972 to 200697 ha in 2016, an increase of $22 \%$. In addition, the bare spaces which were only 666 ha in 1972 moved to 4189 ha, an increase of more than $500 \%$. Finally, the number of semi-permanent pools rose from 219 to 833 from 1972 to 2016, while the number of Koris increased from 280 to 1573 during the same period, an increase of more than $400 \%$. It seems necessary to take urgent measures to safeguard the ecosystems of the region to allow a more balanced development of the area.
\end{abstract}

Keywords: Dallol Bosso, Filingué, Balleyara, Satellite image, Land cover 


\section{Résumé}

Les départements de Filingué et Balleyara, zone de l'étude, sont localisés dans la partie Nord du Dallol Bosso, région de Tillabéri à l'Ouest du Niger. Cette zone d'étude est circonscrite entre $13^{\circ} 35^{\prime}$ et $14^{\circ} 40^{\prime}$ de latitudes Nord et $2^{\circ} 50^{\prime}$ et $3^{\circ} 30^{\prime}$ de longitude Est. Les effets de la variabilité et des changements climatiques survenus dans la région à partir des années 1970 ont eu des impacts sur les écosystèmes. Tenant cela en compte, l'objectif principal de ce travail est d'analyser la dynamique de l'occupation des terres et l'utilisation des sols dans ladite zone. La méthodologie basée sur l'interprétation des imageries satellitaires de 1972, 1987 et 2016, a permis d'aboutir à des résultats importants. Ainsi, on observe une régression notable des superficies occupées par les formations végétales naturelles (brousse tigrée et steppe). En effet, celles-ci passent de 28,79\% en 1972 à 12,15\% en 2016 de la surface totale de la zone d'étude. Au contraire, les terres agricoles sont passées de 164772 ha en 1972 à 200697 ha, soit un accroissement de 22\%. Par ailleurs, les espaces nus qui étaient seulement de 666 ha en 1972 sont passés à 4189 ha, soit une hausse de plus de 500\%. Enfin, le nombre de mares semi permanentes est passé de 219 à 833 de 1972 à 2016, tandis que celui des Koris est passé dans la même période de 280 à 1573 soit une hausse de plus de $400 \%$. Il semble nécessaire de prendre des mesures urgentes pour sauvegarder les écosystèmes de la région afin de permettre un développement plus équilibré de la zone.

Mots-clefs: Dallol Bosso, Filingué, Balleyara, Imageries satellitaires, Occupation des sols

\section{Introduction}

La partie Nord du Dallol Bosso couvre les départements de Filingué et Balleyara, appartenant à la région de Tillabéri dans l'Ouest du Niger. Cette vallée fossile renferme plusieurs potentialités qui attirent de nombreuses populations en quête de conditions favorables à leur survie.

L'objectif principal de cette étude est donc d'évaluer l'évolution de l'occupation des terres et des sols face aux actions anthropiques et aux changements climatiques. De façon plus spécifique, il s'agit d'analyser les différentes imageries satellitaires sur les périodes 1972, 1987 et 2016 afin de quantifier l'évolution des différentes unités d'occupation du sol. Cet article comporte les généralités, le matériel utilisé, la méthodologie, ainsi que les résultats et discussions à ce sujet. 


\section{Cadre de L'étude}

\section{Situation géographique et administrative}

Les départements de Filingué et Balleyara, concernés par cette étude, appartiennent à la région de Tillabéri située à l'Ouest du Niger. Ces deux départements sont limités :

- au Nord par le département d'Abala (région de Tillabéri) ;

- à l' Est par les départements de Doutchi et Loga (région de Dosso) ;

- au Sud par les départements de Kollo (région de Tillabéri) et du Boboye (région de Dosso);

- $\quad$ à l' Ouest par le département de Ouallam (région de Tillabéri).

Située dans le Dallol Bosso, la zone d'étude est circonscrite entre $13^{\circ} 35^{\prime}$ et $14^{\circ} 40^{\prime}$ de latitudes Nord et $2^{\circ} 50^{\prime}$ et $3^{\circ} 30^{\prime}$ de longitude Est (Figure 1). Sa superficie totale est d'environ $2598 \mathrm{~km}^{2}$. Elle couvre partiellement du Nord au Sud 5 communes, dont celles de Filingué, de Kourfeye centre, d'Imanan, de Tondikandia et de Tagazar, appartenant respectivement aux départements de Filingué (les quatre premiers) et à celui de Balleyara (le dernier).

\section{Hydro climatologie}

La zone d'étude est soumise au climat tropical de type sahélien, semiaride, caractérisé par deux saisons bien distinctes, dont une saison pluvieuse de courte durée (juin à septembre) et une saison sèche, de longue durée (octobre à mai). Le régime pluvio-thermique est caractérisé par une pluviosité faible, variable dans le temps et dans l'espace (Mahé et al., 2005), et des températures élevées qui ont tendance à accentuer l'aridité climatique (Alain, 1980). Les hauteurs moyennes annuelles des précipitations observées de 1981 à 2015 au niveau des postes pluviométriques de la zone, indiquent que cellesci diminuent du Sud au Nord, avec des valeurs moyennes annuelles de 440 $\mathrm{mm}$ à Balleyara, $391 \mathrm{~mm}$ à Bonkoukou et $366 \mathrm{~mm}$ à Filingué. Pour la période allant de 2000 à 2015, les températures moyennes mensuelles sont de $12,8^{\circ} \mathrm{C}$ aux mois de décembre et janvier et de $42,7{ }^{\circ} \mathrm{C}$ aux mois d'avril et mai. Pourtant, la température moyenne inter annuelle est de $29,6^{\circ} \mathrm{C}$. Les vents dominants sont le harmattan en saison sèche et la mousson en saison humide. L'évapotranspiration potentielle moyenne annuelle, pour la période considérée, est de $2700 \mathrm{~mm}$, soit près de 7 fois supérieure à la hauteur moyenne inter annuelle de la pluviométrie.

Sur le plan hydrologique, le Dallol Bosso, l'un des principaux affluents de la rive gauche du fleuve Niger, est actuellement fossilisé, et il se caractérise par un endoréisme généralisé. Le réseau hydrographique se résume actuellement en chapelets de mares permanentes ou semi-permanentes, collectant les ruissellements saisonniers intermittents venant des plateaux et/ou des versants. 


\section{Socio économie}

La population vivant dans la zone d'étude, qui était de 150521 habitants en 2012 (RGH/P, 2012 ; INS, 2015), est estimée en 2017 à 171969 habitants, avec une densité moyenne de 76 habitants $/ \mathrm{km}^{2}$.

Les principales activités économiques occupant la presque totalité de la population active dans cette partie de la vallée du Dallol Bosso sont l'agriculture et l'élevage. Ces dernières décennies, les cultures irriguées à partir des nappes alluviales ont connu un développement important. Ainsi, en dehors de la lutte contre l'insécurité alimentaire, elles sont devenues une source de revenus pour les populations. L'élevage, seconde activité économique de la zone, est extensif ou semi-extensif. Néanmoins, le commerce et l'artisanat connaissent une certaine promotion. Enfin, la migration temporaire, vers les grands centres urbains et à l'extérieur du pays, est aussi une source de revenus substantiels pour ces populations.

\section{Morpho pédologie}

La morphologie actuelle est caractérisée par un relief tabulaire et un paysage monotone qui permet toutefois de distinguer schématiquement trois unités géomorphologiques (Courault et al., 1990): (i) les plateaux latéritiques recouverts par des cuirasses ferrugineuses datées du Pliocène, entaillées au cours des différentes périodes pluvieuses du Quaternaire par de larges vallées aujourd'hui comblées en partie par des dépôts sableux. À la limite de ces plateaux, une zone de forte pente (talus) permet de faire la transition avec les glacis sableux ; (ii) les glacis sableux de pente faible ( 2 à $5 \%$ ), sont des dépôts éoliens datant du Pléistocène supérieur ; (iii) les vallées et les bas-fonds dont la pente moyenne nord-sud est de l'ordre $0,18 \%$ o (Tirat, 1964). Concernant les altitudes de la zone d'étude, elles varient de $212 \mathrm{~m}$ à $236 \mathrm{~m}$ au nord du 14 ème parallèle, tandis qu'au sud elles sont comprises entre 167 et $207 \mathrm{~m}$ (Massuel, 2005). Les trois ensembles morpho pédologiques de la zone définis à partir des travaux de plusieurs auteurs (Gavaud, 1966, 1975; Courault et al., 1990, 1991; Nagumo, 1992 ; Desconnets, 1994 ; Ambouta, 1996; d'Herbès \& Valentin, 1997; INRAN-CERRA, 2000) sont :

- les sols peu évolués de type lithosols plus ou moins rubéfiés sur les plateaux latéritiques à cuirasses ferrugineuses ;

- les sables fins et de profil peu différencié sur les glacis sableux ;

- Les sols de type ferrugineux peu lessivés dans les vallées et les basfonds.

\section{Végétation et faune}

La végétation est constituée de fourrésà Combretum sur les plateaux latéritiques ferrugineux du continental terminal. Sur les versants sableux on trouve une végétation clairsemée, colonisée souvent par des savanes herbeuses 
ou arbustives constituées de Guiera senegalensis et Commiphora africana qui peuvent être associées à des espèces ligneuses arborées comme le Faidherbia albida ou acacia et le Balanites aegyptiaca. Une grande diversité d'herbacées, dont des graminées, Aristida mutabilis, Zornia glochidiata, Mitracarpus scaber et Cenchrus biflorus, forme l'essentiel de la strate herbacée annuelle (D'Herbès et al., 1995 in Leduc et Loireau, 1997).

Dans les bas-fonds argileux à sols hydromorphes, la végétation s'organise en fourrés denses plus ou moins dégradés, composés par les Acacia nilotica, Acacia seyal, landes à buissons de Bergia suffruticosa, Bauhinia rufescens et Piliostigma reticulatum (Massuel, 2005). Par ailleurs, dans les zones de stagnation temporaire d'eau, une végétation plus dense et diversifiée se développe (Desconnets, 1994).

Concernant la faune, elle est représentée actuellement par les lapins, les écureuils, les lièvres, les pigeons, les pintades, les varans de terre, les boas et d'autres reptiles. Par ailleurs, on rencontre exceptionnellement quelques gazelles et des girafes.

\section{Géologie et hydrogéologie}

La géologie de cette partie de la vallée du Dallol Bosso comprend essentiellement des sédiments continentaux détritiques, provenant de l'érosion du Continental Intercalaire/Hamadien $(\mathrm{CI} / \mathrm{CH})$, et du Continental Terminal. Ces dépôts constitués par des grés, des sables grossiers et moyens ainsi que des argiles, sont recouverts par des alluvions et/ou par des sables. Sur le plan hydrogéologique, on rencontre de bas en haut: (i) le système aquifère du Continental intercalaire/Hamadien $(\mathrm{CI} / \mathrm{CH})$ qui est captif et artésien par endroit ; (ii) le système aquifère multicouches du Continental Terminal (CT1, CT2, CT3) dont les deux premiers sont captifs et le CT3 libre ; (iii) l'aquifère libre des alluvions, d'extension discontinue. Cette nappe alluviale, qui est affleurante par endroit, est captée à des profondeurs variant de $1 \mathrm{~m}$ en aval dans les zones dépressionnaires à $30 \mathrm{~m}$ dans le secteur amont et sur les plateaux. Les transmissivités sont variables avec une moyenne de l'ordre de $10^{-2} \mathrm{~m}^{2} / \mathrm{s}$. Les eaux de cette nappe sont relativement peu minéralisées et douces, mais elles sont très sensibles aux pollutions chimiques et/ou bactériologiques. Leur température moyenne est de l'ordre de $30^{\circ} \mathrm{C}$. 




Figure 1. Présentation de la zone d'étude

\section{Matériel et Méthodes \\ Matériel}

Données

Le matériel utilisé est constitué par des données et des outils :

Les données, essentiellement collectées au Centre Régional AGRHYMET, comprennent :

1. Les données ancillaires de référence constituées par :

- Trois (3) cartes topographiques IGN (échelle 1 : 200 000);

$>$ Feuille ND 31 XVI (FILINGUE)

$>$ Feuille ND $31 \mathrm{X}$ (DOSSO)

$>$ Feuille ND 31 IX (NIAMEY) 
- Une (1) carte d'occupation et utilisation des terres en 2013 du Niger, programme LULC du Centre Régional AGRHYMET/USGS.

2. Les données de base, constituées d' imageries satellitaires, qui sont :

- Image Landsat 1 Capteur MSS :

- Scène P206R050 du 09 novembre 1972

- Images Landsat 5 Capteur TM :

$>$ Scène P192R050 du 15 octobre 1987

$>$ Scène P192R051du $1^{\text {er }}$ février 1987

- Images Landsat 8 Capteur OLI :

Scène P192R050 du 28 septembre 2016

Scène P192R051 du 28 septembre 2016

- Images Google Earth@ 2017

\section{Outils}

Les outils sont constitués essentiellement par les deux logiciels SIG suivants :

ArcGIS version 10.4.1 : Il a servi notamment pour les traitements d'images et les restitutions cartographiques.

ArcView GIS 3.3 : Il a été utilisé pour la numérisation à l'écran des classes d' utilisation et d' occupation des terres.

\section{Méthodes}

Le procédé d'élaboration des cartes d'occupation des sols utilisé s'effectue en deux étapes :

\section{Prétraitements d'images satellitaires}

Les prétraitements sont des opérations effectuées sur les images en amont de tout traitement:

Corrections géométriques de l'image :

Toutes les images satellitaires utilisées dans le cadre de cette étude sont corrigées par le distributeur. Ainsi, leur correction géométrique n'a pas été nécessaire ;

- Améliorations visuelles des images :

L'objectif recherché dans cette étape est la modification de l'aspect visuel de l'image pour faciliter son interprétation. Ainsi, toutes les images utilisées ont été soumises à des opérations élémentaires d'ajustement de contrastes (rehaussement des luminances) et de lissage (adoucissement des tons de l'image).

* Création d'une mosaïque d'images :

La zone d'étude est couverte par deux scènes d'images Landsat. Cette opération a consisté à juxtaposer les deux scènes pour en former une seule. 
Extraction de la zone d'étude

La zone d'étude a été délimitée et extraite à partir des images mosaïquées.

\section{Traitement et analyse des données}

Une fois les images prétraitées, mosaïquées, et la zone d'étude extraite, on passe aux opérations suivantes :

Composition colorée et interprétation visuelle de l'image :

La composition colorée consiste à combiner des informations contenues dans trois bandes en les affichant simultanément dans les trois couleurs primaires (rouge, vert et bleu). Le but de cette opération est d'avoir une synthèse d'informations permettant d'avoir une bonne discrimination des types d'utilisation et d'occupation des terres.

La composition colorée 432 a été choisie pour les images TM, tandis que pour les images Landsat MSS la composition colorée 421 a été retenue. Quant aux images Landsat 8, c'est la composition colorée 543 celle qui a été utilisée.

\section{Interprétation :}

La bonne connaissance de la zone d'étude a permis d'opter pour une interprétation visuelle. En effet, celle-ci permet l'extraction des zones homogènes par leur texture, leur structure, leur tonalité ou leur agencement dans l'espace. Il s'agit d'une démarche plus ou moins complexe d'analyse et de synthèse conduisant à une reconnaissance plus ou moins stricte des objets sur les photographies ou les images satellitaires. Elle consiste à faire une délimitation pour les zones homogènes et affecter une classe d'occupation pour chacune.

L'interprétation visuelle s'effectue en analysant l'image affichée sur un écran d'ordinateur. Cette opération a consisté à délimiter quelques unités des éléments interprétés sur la base des clés d'interprétation fondées sur les notions de texture et de structure. Des codes numériques sont attribués à chaque type d'unité, afin de la distinguer des autres. A cet effet, une légende provisoire a été établie sur la base du document du MHE/LCD réalisé en 2001, intitulé «Nomenclature pour la construction de Base de Données sur l'Occupation des Sols au Niger au Sud du 16ème Parallèle», et le tableau des réponses spectrales des objets. Ainsi, les classes d'utilisation et d'occupation retenues sont :

1. Cordons rupicoles, ce sont des forêts galeries constituant une bande étroite en bordure du cours d'eau;

2. Brousse tigrée régulière et dégradée, il s'agit des formations végétales contractées, caractérisées par une alternance de bandes de végétation arbustive dense et de bandes nues ; 
3. Steppe, elle est composée par des formations herbeuses ouvertes dominées par des graminées vivaces au sein desquelles se rencontrent arbres et/ou arbustes et des plantes annuelles ;

4. Cultures pluviales continues, qui sont des zones sur lesquelles des cultures sont réalisées au cours de la saison de pluie ;

5. Jachères, elles sont des terres de cultures laissées au repos afin d'en restaurer la fertilité ;

6. Mares semi permanente, il s'agit d'étendues d'eau temporaires ;

7. Kori, ce sont des cours d'eau à écoulement intermittent ;

8. Zone d'habitation, elle comprend des villes et villages ;

9. Sols nus, ce sont des sols dépourvus de couverture végétale, qui regroupent les sols dénudés, des terrains rocheux, des plateaux dénudés, et des dunes.

L'interprétation des images satellitaires traitées constituent la base pour l'analyse de la topographie et de l'occupation des sols de la zone ainsi que de leur évolution. Les produits ainsi obtenus sont :

- une carte du Modèle Numérique de Terrain (MNT) de la zone ;

- les cartes décrivant l'occupation des sols en 1972, 1987 et 2016 ;

- les tableaux des états des unités d'occupation des sols pour 1972, 1987 et 2016.

L'évolution d'utilisation et d'occupation des terres est appréciée sur la base d'une analyse comparative de modification subie par les classes suivant un pas de temps (1972-1987) et (1987 - 2016). Par ailleurs, les changements globaux ont été quantifiés et analysés selon la variation des superficies de départ (1972) et d'arrivée (2016), pour chaque classe d'utilisation et d'occupation des terres.

\section{Résultats et Discussions}

\section{États des occupations des terres et des sols en $1972 ; 1987 ; 2016$} État en 1972

La superficie totale de la zone étudiée est d'environ 257304 ha. La carte d'utilisation et d'occupation des terres de 1972 (Figure 2), montre que la zone d'étude présentait déjà des signes de saturation pour les cultures pluviales qui occupaient 164772 ha, soit 64\% de sa superficie totale. Toutefois, on observe une couverture végétale appréciable. En effet, les brousses tigrées (régulière et dégradée) et la steppe couvraient respectivement 27400 et 43242 ha, soit environ 11 et $17 \%$ de la superficie totale, tandis que les sols dénudés ne représentaient que 666 ha, soit $0,26 \%$. Les zones d'habitation occupaient 1280 ha. Le nombre de mares semi permanentes sont estimées à 219 et les Kori à 280 (Tableau.1). 
Tableau 1. Superficies des classes d'utilisation et d'occupation des terres en 1972

\begin{tabular}{|l|r|r|}
\hline Classes d'utilisation et d'occupation des terres & Superficie (ha) & Superficie (\%) \\
\hline Zone d'habitation (ZH) & 1280 & 0.50 \\
\cline { 1 - 1 } Mare semi permanente(MSP) & 219 & 0.09 \\
Kori (KO) & 280 & 0.11 \\
\hline Sols dénudés (SD) & 666 & 0.26 \\
Steppe (SP) & 43242 & 16.81 \\
\hline Cultures pluviales en continue (CPC) & 164772 & 64.04 \\
\hline Terrain rocheux/Plateau dénudé (TR) & 8513 & 3.31 \\
\hline Jachères (JA) & 7479 & 2.91 \\
\hline Brousse tigrée régulière (BTR) & 6630 & 2.58 \\
\hline Cordons rupicoles (CR) & 3435 & 1.33 \\
\hline Brousse tigrée dégradée (BTD) & 20770 & 8.07 \\
\hline TOTAL & $\mathbf{2 5 7 3 0 4}$ & $\mathbf{1 0 0 . 0 0}$ \\
\hline
\end{tabular}

État d'occupation des terres en 1987

En 1987, les surfaces occupées par les cultures pluviales sont estimées à 186264 ha, soit 72\% de la superficie de la zone d'étude, tandis que la couverture végétale, représentée par les brousses tigrées (régulière et dégradée) et la Steppe occupent respectivement 24855 ha, soit moins de 10\% et 24839 ha, soit environ $9,65 \%$. Par contre, les sols dénudés représentent 881 ha, soit $0,34 \%$ de la superficie totale. Le nombre de plans d'eau temporaires et les Koris sont estimés respectivement à 418 et 468 . En ce qui concerne les zones d'habitations, elles sont de 1936 ha, soit $0,75 \%$ de la superficie totale (Tableau 2).

Tableau 2. Superficies des classes d'utilisation et d'occupation des terres en 1987

\begin{tabular}{|l|c|c|}
\hline Classes d'utilisation et d'occupation des terres & Superficie (ha) & Superficie (\%) \\
\hline Zone d'habitation (ZH) & 1936 & 0.75 \\
\cline { 1 - 1 } Mare semi permanente (MSP) & 418 & 0.16 \\
\cline { 1 - 1 } Kori (KO) & 468 & 0.18 \\
\cline { 1 - 1 } Sols dénudés (SD) & 2481 & 0.34 \\
\cline { 1 - 1 } Steppe (SP) & 186264 & 9.65 \\
\cline { 1 - 1 } Cultures pluviales en continue (CPC) & 9296 & 72.39 \\
\cline { 1 - 1 } Terrain rocheux/Plateau dénudé (TR) & 6228 & 3.61 \\
\cline { 1 - 1 } Jachères (JA) & 7552 & 2.42 \\
\cline { 1 - 1 } Brousse tigrée régulière (BTR) & 2119 & 2.98 \\
\cline { 1 - 1 } Cordons rupicoles (CR) & 17303 & 0.82 \\
\hline Brousse tigrée dégradée (BTD) & $\mathbf{2 5 7 3 0 4}$ & $\mathbf{1 0 0 . 0 0}$ \\
\hline TOTAL & &
\end{tabular}

État d'occupation des terres en 2016

Les zones de cultures pluviales sont de 200687 ha, soit $78 \%$ de la superficie totale. La couverture végétale représente environ 17665 ha, soit 
moins de 7\% de la superficie totale. Concernant les surfaces occupées par la steppe, les sols dénudés et les zones d'habitations, elles sont respectivement de 7770,4189 et 3548 ha, soit 3\%, 1,63\% et 1,38\%. Le nombre des mares semi permanentes est de 833, tandis que celui des Koris s'élève à 1572 (Tableau 3).

Tableau 3. Superficies des classes d'utilisation et d'occupation des terres en 2016

\begin{tabular}{|l|c|c|}
\hline Classes d'utilisation et d'occupation des terres & Superficie (ha) & Superficie (\%) \\
\cline { 1 - 1 } Zone d'habitation (ZH) & 3548 & 1.38 \\
\cline { 1 - 1 } Mare semi permanente (MSP) & 833 & 0.32 \\
\cline { 1 - 1 } Kori (KO) & 1572 & 0.61 \\
\hline Sols dénudés (SD) & 4189 & 1.63 \\
\cline { 1 - 1 } Steppe (SP) & 7770 & 3.02 \\
\hline Cultures pluviales en continue (CPC) & 200697 & 78.00 \\
\hline Terrain rocheux/Plateau dénudé (TR) & 10044 & 3.90 \\
\hline Jachères (JA) & 5159 & 2.01 \\
\hline Brousse tigrée régulière (BTR) & 489 & 0.19 \\
\hline Cordons rupicoles (CR) & 5818 & 2.26 \\
\hline Brousse tigrée dégradée (BTD) & 17184 & 6.68 \\
\hline TOTAL & $\mathbf{2 5 7 3 0 4}$ & $\mathbf{1 0 0 . 0 0}$ \\
& & \\
& & \\
& & \\
\end{tabular}




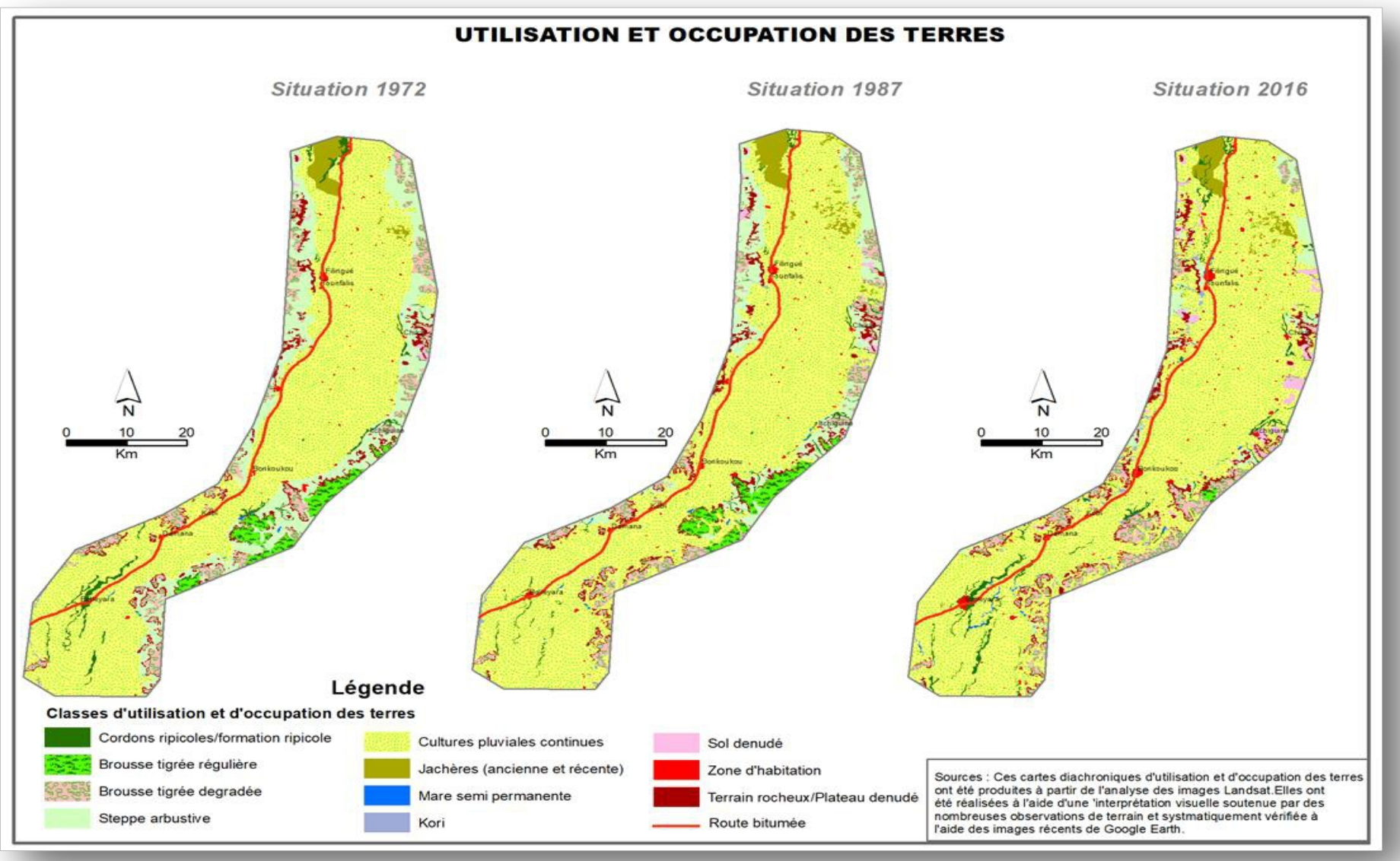

Figure 2. Carte d'utilisation et d'occupation des terres en $1972 ; 1987$ et 2016 


\section{Évolution de l'occupation des sols}

L'évolution de l'occupation des sols au cours des années successives 1972-1987 et 1987-2016, permet d'observer les faits suivants :

\section{Évolution de l'occupation des terres entre 1972 et 1987}

L'évolution des unités d'occupation des sols entre 1972 et 1987 montre que les superficies occupées par les cultures pluviales en continu ont connu une progression de 13\%, passant de 164772 ha à 186264 ha, au détriment des deux autres classes majoritaires qui sont les brousses tigrées dégradés et des zones de Steppes, dont les aires ont baissé respectivement de 17 et $43 \%$ (fig. 3 ). Au niveau des classes intermédiaires, les champs de jachères et les cordons ripicoles ont vu leurs surfaces réduites respectivement de 17 et $38 \%$, contrairement aux terrains rocheux/plateaux dénudés et à la brousse tigrée régulière lesquels ont connu des progressions respectives de 9 et $14 \%$ (Figure 4). Concernant les classes minoritaires, les zones d'habitation sont passées de 1280 ha à 1936 ha, soit une hausse de 51\%, traduisant ainsi un accroissement considérable des populations dans la zone. Leurs activités anthropiques pourraient expliquer cette dégradation des terrains naturels (brousses, steppes) au profit des zones agricoles. De même, la superficie des sols nus a connu une progression, passant de 666 à 881 ha. Enfin, concernant les Koris et les mares semi permanentes, leur nombre a passé respectivement de 280 à 468 et de 219 à 418.

\section{Évolution de l'occupation des terres 1987 à 2016}

Au niveau des formations végétales naturelles de la classe majoritaire, la régression de la superficie occupée par les brousses tigrées dégradées reste faible, voire négligeable. Par contre, au niveau des zones de steppe, cette diminution est très élevée. En effet, de 24839 ha, elle tombe à 7770 ha, soit une baisse de $69 \%$ au profit des zones de culture pluviales en continue, ayant augmenté de $11 \%$ (Figure 3). Concernant les classes intermédiaires, les aires des terrains rocheux/plateau dénudés et des cordons rupicoles ont progressé respectivement de 8 et $174 \%$ contrairement à celles occupées par les champs en jachères et les brousses tigrées régulières qui ont baissé de 17 et $94 \%$ (Figure 4). Enfin, à propos de la classe minoritaire, les zones d'habitation ont passé dans la même période de 1936 à 3548 ha, soit une hausse de 82\%, traduisant une forte pression démographique susceptible de provoquer une dégradation des terrains naturels. Ainsi, les sols dénudés estimés à 881 ha en 1987, occupent en 2016 une superficie de 4189 ha, soit une progression de $78 \%$. L'accentuation du ruissellement de surface est l'une des principales conséquences de ce phénomène. En effet, on observe une augmentation importante du nombre de Koris et de mares semi permanentes, passant respectivement de 468 à 1572 et 418 à 833 entre 1987 et 2016, soit des hausses 
de 235 et de $100 \%$. Ces résultats sont conformes à ceux de certains auteurs (Albergel, 1987 ; Amani \& Nguétora, 2002).

Évolution de l'occupation des terres 1972 à 2016

Les différentes unités d'occupation des sols montrent une évolution importante entre 1972 et 2016 (Figure 2). En effet, la classe cultures pluviales continues a enregistré une hausse de $22 \%$; de même, les superficies des terrains rocheux, les dunes et les sols dénudés se sont accrues considérablement. Ainsi, les sols nus ont passé de 666 à 4189 ha, contribuant à l'accentuation de l'érosion hydrique et éolienne. Par contre, les aires des formations végétales naturelles (cordons ripicoles, brousse tigré dégradée, brousse tigré régulière et steppe), ont diminué de plus de leur moitié au cours de cette période, en passant de $28,79 \%$ à $12,15 \%$ de la surface totale de la zone d'étude. Pour le cas spécifique de la steppe, on constate que sa superficie, qui représentait près de $17 \%$, est passée à 3,02\% de l'aire totale de la zone. Par ailleurs, le nombre de Koris et des mares semi permanentes a connu une progression très importante en passant respectivement de 280 à 1572 et de 219 à 233.

De manière générale, un processus de dégradation généralisée des terres est observé dans la zone d'étude. Celle-ci touchant principalement les unités relatives aux brousses et aux steppes, pourrait s'expliquer par les actions anthropiques (extension des zones de cultures, élevage extensif, coupes de bois de chauffe, culture sur brulis, artisanat...etc.), mais aussi par les variabilités et changements climatiques survenus dans la zone.

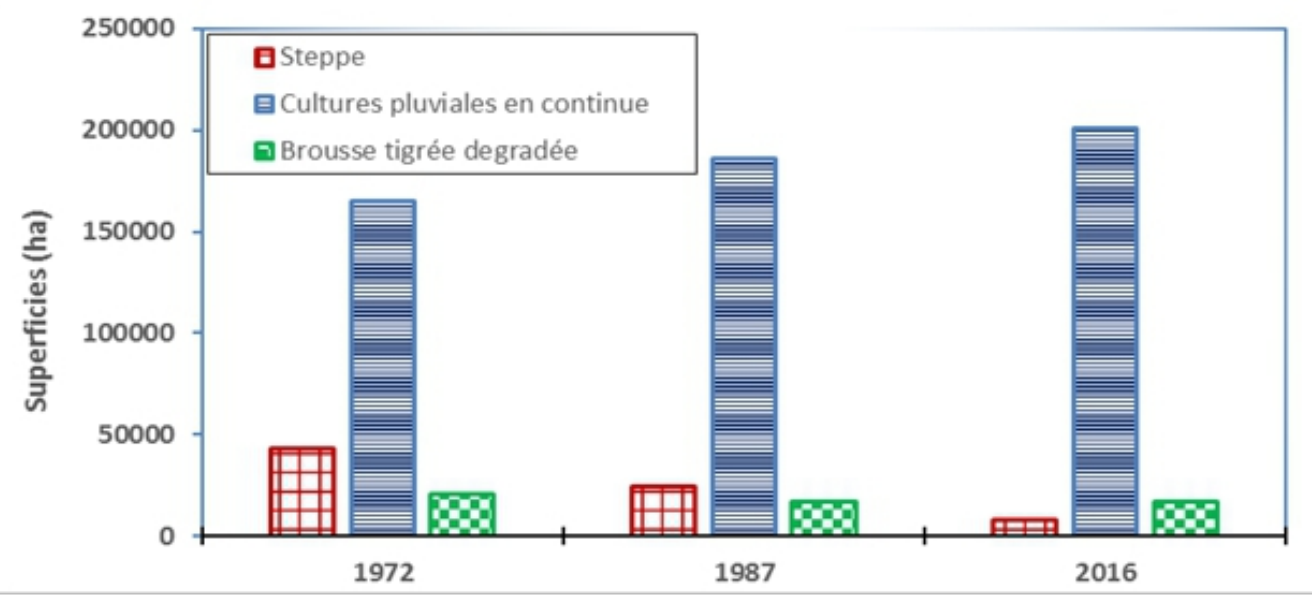

Figure 3. Évolution des superficies des classes majoritaires au cours de trois périodes 


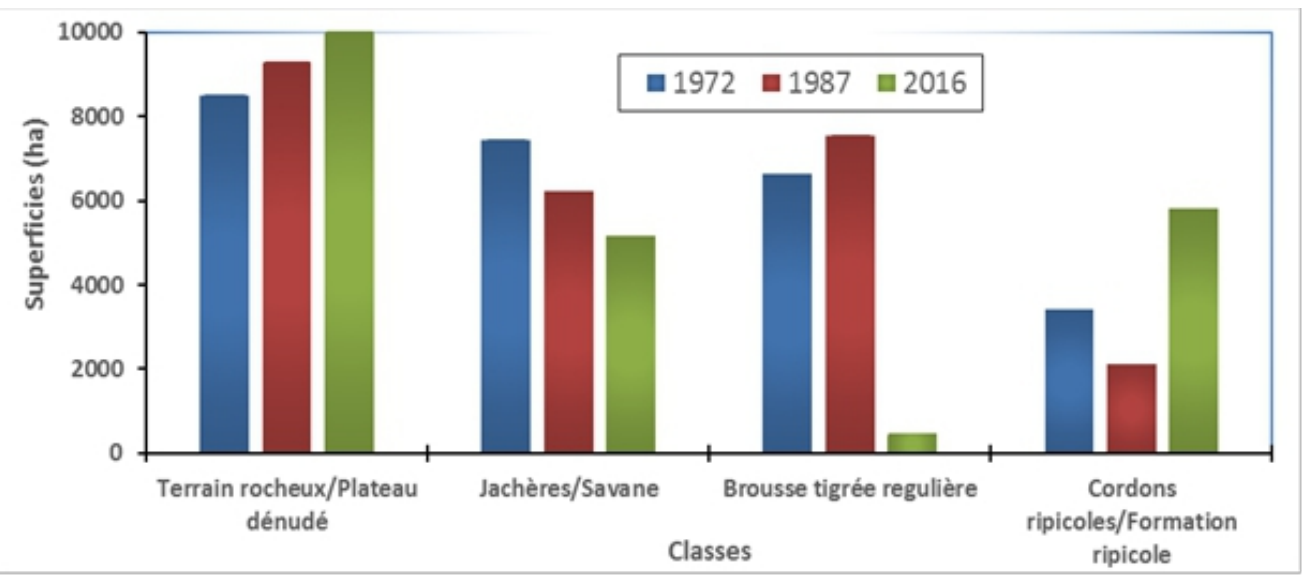

Figure 4. Évolution des superficies des classes intermédiaires au cours de trois périodes

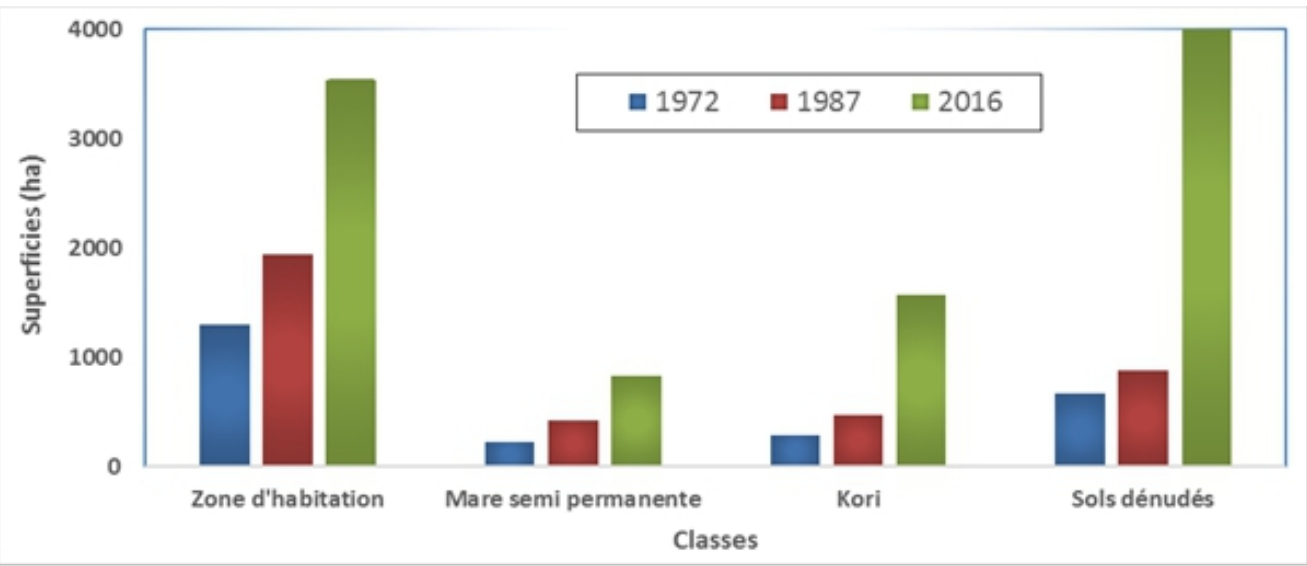

Figure 5. Évolution des superficies des classes minoritaires au cours de trois périodes

\section{Conclusion}

L'analyse de l'évolution de l'état d'occupation du sol dans la zone d'étude de 1972 à 2016 a permis de montrer que la zone a subi une dégradation généralisée de son écosystème, se traduisant par une régression importante des formations végétales naturelles (brousses tigrées et steppes) au profit des paysages aménagés et des sols dénudés. L'extension des zones d'habitation, la pression démographique, les changements des comportements des populations à travers l'utilisation de nouvelles pratiques destructrices de l'environnement, associés aux facteurs climatiques, semblent être à la base de ce phénomène de la dégradation des sols. Il s'avère important de mener des actions de restauration et de récupération des sols dans ces vallées et aussi de sensibiliser les populations sur les conséquences de certaines pratiques. 


\section{References:}

1. Alain, M. (1980). Les Atlas Jeune Afrique : Atlas du Niger, Edition Jeune Afrique Paris 64 pages : 6-21.

2. Albergel, J. (1987). Sécheresse, désertification et ressources en eau de surface : application aux petits bassins du Burkina Faso. In The Influence of Climate Change and Climatic Variability on the Hydrologic Regime and Water Resources; IAHS publication $\mathrm{N}^{\circ} 168$, Wallingford, UK, pp. 355-365.

3. Amani, A. \& Nguetora, M. (2002). Evidence d'une modification du régime hydrologique du fleuve Niger à Niamey. In FRIEND 2002 Regional Hydrology: Bridging the Gap between Research and Practice, Proceedings of the Friend Conference, Cape Town, South Africa, 18-22 March, 2002; Van Lannen, H., Demuth, S., Eds.; IAHS publication $\mathrm{N}^{\circ} 274$, Wallingford, UK, pp. 449-456.

4. Ambouta, K., Laverdiere, M.R., \& Valentin, C. (1996). Jachères et croûtes d'érosion au Sahel. Sec., n.4, 7, 269-275.

5. Courault, D., D'herbes, L.M., \& Valentin, C. (1990). Le bassin versant de Sama Dey, Premières observations pédologiques et phytoécologiques. Rapport multigraphié, ORSTOM 37p.

6. Courault, D., D'herbes Jean-Marc, Valentin Christian \& Remy Dominique (1991). Etude de la variabilité spatiale du comportement hydrodynamique des sols au Niger à partir d'images satellitaires. In : Physical measurements and signatures in remote sensing. Noordwijk: ESA, (319), 601-604. (ESA SP; 319). International Colloquium. Physical Measurements and Signatures in Remote Sensing, 5., Courchevel (FRA), 1991/01/14-18.

7. Desconnets, J.C. (1994). Typologie et caractérisation hydrologique des systèmes endoréiques en milieu sahélien. (Niger-Degré carré de Niamey). Thèse sciences, 326 p. Université de Montpellier II, France.

8. D'herbes, J.M. \& Valentin. C. (1997). Land surface conditions of the Niamey region: ecological and hydrological implications. Journal of Hydrology, Volumes 188-189, Pages 18-4.

9. D'herbes, J.M., Valentin, C., \& Thiery, J.M. (1997). La brousse tigrée au Niger: synthèses des connaissances acquises. Hypothèses sur la genèse et les facteurs déterminants les différentes structures contractées. John Libbey Eurotext, Paris-1997, pp 131-152.

10. Gavaud, M. (1977). Les grands traits de la pédogénèse au Niger méridional. Trav. dot. ORSTOM 76, 102 p, Paris, France.

11. Inran-Cerra (2000). Etude pédologique des sites du projet pilote de promotion de l'irrigation privée. 
12. INS-Niger (2015). Tillabéry en chiffre. Document de la direction régionale de l'INS Tillabéry, du Ministère de l'Economie et des Finances, Edition.

13. Institut National de la Statistique (2014). Recensement Général de la Population et de l'Habitat (RGP/H) de 2012. Ministère des Finances, Niger, 4ème Série, 24 pages.

14. Leduc, C. \& Loireau, M. (1997). Fluctuations piézométriques et évolution du couvert végétal en zone sahélienne (sud-ouest du Niger). in: "Sustainability of water resources under increasing uncertainty", Rabat symposium Sl, 1997. AZSH Publ., 240, 193-200.

15. Mahe, G. \& Olivry J-C. (1995). Variations des précipitations et des écoulements en Afrique de l'Ouest et centrale de 1951 à 1989. Sécheresse.vol.6, $\mathrm{n}^{\circ} 1,109-117$.

16. Massuel, S. (2005). Evolution récente de la ressource en eau consécutive aux changements climatiques et environnementaux du sud-ouest Niger Modélisation des eaux de surface et souterraines du bassin du kori de Dantiandou sur la période 1992-2003. Thèse de doctorat, UNIVERSITE MONTPELLIER II, Montpellier (France), 220 pages ;

17. Nagumo, F. (1992). Topographical map and soil map at the central site of the sahelian HAPEX project in Niger. Rapport multigraphié, 22p.

18. Tirat, M. (1964). Contribution à l'étude hydrogéologique du continental terminal. Rapport BRGM, NIA. 64. Ai, 77p. Niamey, Niger.- Orléans : BRGM, - Projet NIA. 64. A1. 